Midori Asada-Senju • Toyoki Maeda • Toshiie Sakata Akira Hayashi • Tomokazu Suzuki

\title{
Molecular analysis of the transferrin gene in a patient with hereditary hypotransferrinemia
}

\begin{abstract}
We previously reported a patient with hereditary hypotransferrinemia who suffered from severe anemia and growth retardation and was diagnosed on the basis of an extremely low level of serum transferrin (TF). By an isoelectric focusing analysis, we found that the patient and his father shared a variant TF protein with an abnormal isoelectric point. The study suggested that the patient was a compound heterozygote with a variant allele, encoding the mutant TF, of paternal origin and a null allele of maternal origin. In the present study, we investigated the $T F$ gene of the patient and his family. We showed that the patient and his father shared a variant $T F$ gene bearing a GAA to AAA transition at codon 394. This nucleotide substitution causes a nonconservative amino acid change from glutamate to lysine in amino acid residue 375 of the TF protein. This single amino acid mutation is predicted to cause a conformational change in the coiled region of the carboxylterminal iron-binding lobe. As for the maternal null allele, no mutation was found in either the coding region or the exon-intron boundaries, suggesting an abnormality in the transcription or stability of mRNA of maternal allele origin.
\end{abstract}

Key words Transferrin - Familial hypotransferrinemia • Gene analysis $\cdot$ Mutation $\cdot$ PCR-RFLP

M. Asada-Senju $\cdot$ T. Maeda $(\bowtie) \cdot$ T. Suzuki ${ }^{1}$

Department of Clinical Genetics, Medical Institute of Bioregulation,

Kyushu University, 4546 Tsurumihara, Beppu, Oita 874-0838, Japan

Tel. +81-977-27-1670; Fax +81-977-27-1671

e-mail:maeda@tsurumi.beppu.kyushu-u.ac.jp

M. Asada-Senju · T. Sakata

Department of Internal Medicine I, School of Medicine, Oita Medical University, Oita, Japan

A. Hayashi

Division of Cancer Immunotherapy, Osaka Medical Center for

Cancer and Cardiovascular Disease, Osaka, Japan

Present address:

${ }^{1}$ Kinki Central Hospital, Itami, Japan

\section{Introduction}

Iron is an essential metal required by most living organisms. Because iron is only slightly soluble under physiological $\mathrm{pH}$ and ionic conditions, organisms have been compelled to evolve iron-binding proteins to facilitate its transport and storage. In vertebrates, transferrin (TF), a major plasma protein of biological interest, plays a significant physiological role in the transport of iron among the sites of absorption, storage, and utilization. TF also plays an important role in bacteriostasis, cell proliferation, and heme synthesis in reticulocytes (Morgan 1983; Ponka and Schulman 1985).

The synthesis and storage of TF are regulated by levels of iron and estrogen and by nutritional status (Morgan 1983). TF transports ferric iron into cells by receptormediated endocytosis, a unique process by which TF and its receptor are reutilized repeatedly for iron delivery. In humans, the serum TF concentration decreases during iron overload and increases during chronic iron deficiency. TF is primarily synthesized in the liver, but significant amounts are also produced in the brain, testis, and lactating mammary gland, and in some fetal tissues during development. The $T F$ gene was previously cloned, characterized, and mapped to human chromosome 3, within the region of 3q21-25 (Yang et al. 1984).

Atransferrinemia (Familial hypotransferrinemia, MIM 209300) is a very rare human disorder. Only nine patients in eight families have been reported since 1961 (Heilmeyer et al. 1961; Cáp et al. 1968; Sakata 1969; Walbaum 1971; Goya et al. 1972; Loperena et al. 1974; Hamil et al. 1991; Beutler E et al. 2000). We previously analyzed the TF protein of the patient reported by Goya et al. (1972) and his family, and concluded that the patient and his two healthy siblings were compound heterozygotes with a paternal "variant" allele and a maternal "null" allele (Hayashi et al. 1993).

In the present study, to determine the molecular basis of his atransterrinemia, we analyzed the nucleotide sequence of the entire coding region of the $T F$ gene of this patient and his family. As a result, we found a missense mutation com- 
mon to the patient and his father at codon 394 of the $T F$ gene.

\section{Subjects and methods}

\section{Subjects}

The proband was originally reported as having congenital atransferrinemeia (Goya et al. 1972). Only a trace of transferrin was detected in his plasma by a turbidimetric immunoassay (Ritchie 1975; Hayashi et al. 1993). Supplementary therapy with apo-TF alleviated the anemia. An isoelectric focusing analysis revealed a shifted TF variant, which was detected in all three siblings as well as in their father.

\section{DNA preparation}

With informed consent, peripheral blood from the patient and his parents and umbilical cord blood of the proband's niece, a daughter of the patient's sister, were collected. Blood samples were also obtained from 100 normal Japanese volunteers. Genomic DNA was thereafter prepared from leukocytes by using a standard method.

Amplification of the $T F$ gene using polymerase chain reaction and long accurate polymerase chain reaction analyses

Genomic DNA fragments spanning one or two exons of the $T F$ gene were amplified by polymerase chain reaction (PCR) and then subjected to a sequence analysis. PCR was performed by using $100 \mathrm{ng}$ genomic DNA, the primers, $0.2 \mathrm{mM}$ of each dNTP, 0.5 U Taq DNA polymerase (Wako, Osaka, Japan) in the presence of $10 \mathrm{mM}$ Tris $\mathrm{HCl}, \mathrm{pH} 8.3$, $50 \mathrm{mM} \mathrm{KCl}, 1.5 \mathrm{mM} \mathrm{MgCl}_{2}$, and $0.1 \%$ Triton X-100 to a final volume of $10 \mu \mathrm{l}$ for 30 cycles as follows: $1 \mathrm{~min}$ at $94^{\circ} \mathrm{C}, 1 \mathrm{~min}$ at $55^{\circ} \mathrm{C}$, and $1 \mathrm{~min}$ at $72^{\circ} \mathrm{C}$. In some analyses, long accurate (LA)-PCR was performed by using $500 \mathrm{ng}$ genomic DNA, the primers, a dNTP mixture at $0.4 \mathrm{mM}$ each, $0.5 \mathrm{U}$ LATaq DNA polymerase (Takara, Tokyo, Japan) in the presence of $10 \times$ LA-PCR buffer to a final volume of $50 \mu \mathrm{l}$ for $1 \mathrm{~min}$ at $94^{\circ} \mathrm{C}, 14$ cycles of $20 \mathrm{~s}$ at $98^{\circ} \mathrm{C}$ followed by $20 \mathrm{~min}$ at $68^{\circ} \mathrm{C}$, and then 16 cycles as follows: $20 \mathrm{~s}$ at $98^{\circ} \mathrm{C}, 20 \mathrm{~min}$ and $15 \mathrm{~s}$ at $68^{\circ} \mathrm{C}$, and $10 \mathrm{~min}$ at $72^{\circ} \mathrm{C}$. Some PCR products were purified and inserted into the plasmid vector pGEM-T (Promega, Madison, WI, USA).

\section{PCR primers}

Oligonucleotide primers were synthesized based on the nucleotide sequence of the $T F$ gene. The primers used for amplification of each of the exons are shown in Table 1.
DNA sequence analysis

The exonic regions of the $T F$ gene from the proband and his parents containing a variant sequence were subjected to a direct sequencing analysis by using Sequenase version 2.0 (United States Biochemical, Cleveland, OH, USA), $\left[\alpha-{ }^{32} \mathrm{P}\right]$ deoxy cytosine triphosphate (dCTP), and a Sequencing PRO (Toyobo, Osaka, Japan) sequencing kit. Some PCR fragments were inserted into a pGEM-T vector, and multiple independent subclones were sequenced. The samples were run for $1.5-3.0 \mathrm{~h}$ in a $6 \%$ polyacrylamide denaturing gel in $0.5 \times \mathrm{TBE}$ (Tris borate + ethylenediaminetetraacetate) buffer, pH 8.0, and processed by using BAS 1000 (Fuji Film, Tokyo, Japan).

PCR-restriction fragment length polymorphism analysis

The patient and his father were characterized with respect to the mutated genotype of the $T F$ gene. MboII digestion of the PCR product amplified by using primers TFL93 (5'AGAGGCTCAAGTGTGATGAGTGGAGTGTTA-3') and TF 92i (5'-GACGCTGCCCTAGGAAGGCTG-3') was performed to detect the Glu375-to-Lys mutation in the variant allele. An identified mutation of the proband located at an MboII site was tested for by using restriction fragment length polymorphism (RFLP) in the proband's family and in healthy control individuals.

Prediction of the secondary structure of the TF protein

Secondary structure prediction was performed by the method of Chou and Fasman (1978) by using DNAsis version 3.6.1 (Hitachi Software Engineering, Yokohama, Japan).

\section{Results}

We could not analyze the TF cDNA of the patient, because it is practically impossible to obtain RNA samples from the tissues where TF is expressed, such as liver and brain. Therefore, we amplified genomic DNA fragments by using $\mathrm{PCR}$, covering the entire protein-coding region and the exon-intron boundaries, to examine the $T F$ gene of the patient with hypotransferrinemia. Some PCR fragments were subjected to direct sequencing, while others were inserted into a plasmid vector and multiple independent plasmid clones were sequenced. We also analyzed the $T F$ gene of the patient's parents. The nucleotide sequences of the $T F$ genes of the patient and his parents were compared with two previously reported cDNA sequences (Table 2) (Yang et al. 1984; Park et al. 1985; Schaeffer et al. 1987).

For codon 247 in exon 7 , encoding amino acid residue 228 of the mature TF protein, two different sequences, CTA (Yang et al. 1984) and CTG (Park et al. 1985), have been previously reported. The patient's father was homozygous for CTG. On the other hand, the patient and his mother 
Table 1. Polymerase chain reaction primers

Exon 2

Exon 5

Exon 7

Exon 8

Exon 9

Exon 12

Exon 15

Exon 16

Exon 17

Exons 1 and 2

Exons 3 and 4

Exons 4 and 5

Exons 5 and 6

Exons 6 and 7

Exons 7 and 8

Exons 8 and 9

Exons 10 and 11

Exons 10, 11 and 12

Exons 12 and 13

Exons 15 and 16
TF21: 5'-GGCTGTGTCTGGCTGTC-3'

TF22: 5'-CGCAATGGCCCTGATGC-3'

TF51: 5'-CAGTGGCCAATTTCTTC-3'

TF52: 5' -TTGAAGGCTCCCGAG-3'

TF71: 5'-AGAACTTGGCAAACAAG-3'

TF72: 5'-CTGGGCCTGGTTGAGAAG-3'

TF81: 5' -CAGGAACATTTTGGCAAA-3'

TF82: 5'-ATGTGCCTTCCCGTAG-3'

TF91: 5'-CCAGAAGCCCCAACAGAT-3'

TF92: 5'-CATGATCTTGGCGATGCA-3'

TF121: 5'-GGTATTTTGCTGTAGC-3'

TF122: 5'-CAAATCTGCAGTGGTTGA-3'

TF151: 5'-GAAAAAACCCTGATCCA-3'

TF152: 5'-CTGCTGTTGACGTAATAT-3'

TF161: 5'-CACCTATTTGGAAGC-3'

TF162: 5'-ATGAGGTGGAGCATTTTC-3'

TF171: 5'-CACTCCTGGAAGCCTGCA-3'

TF174: 5'-GCACACATGGCAACACAG-3'

TF501: 5'-CCCGCCCAGGCCGGGAATGGAATAAAGGGA-3'

TF24: 5'-CTTTTCATATGGTCGCGGAAACTCTGGCAC-3'

TF31: 5'-GCAAACGAAGCGGATGC-3'

TF44: 5'-CTTACCTTTCTCAAGAGGTTTAC-3'

TFL43: 5'-ACCAGCTTCGAGGCAAGAAGTCCTGCCACA-3'

TFL54: 5'-AGCCACACCCTGGACACAGTTGACACAGCT-3'

TF51: 5'-CAGTGGCCAATTTCTTC-3'

TFL64: 5'-CAAATATAGTCGAGTGCTTGACAAAGGCCACATCCC-3'

TF61: 5'-GTGTCTGAAGGATGGTGC-3'

TF74: 5'-CTGGGCCTGGTTGAGAAGCTC-3'

TF75: 5'-CTTGGCAAACAAGGCTGACAG-3'

TF82: 5'-ATGTGCCTTCCCGTAG-3'

TF83: 5'-GAACATTTTGGCAAAGACAAA-3'

TF94: 5'-CATGATCTTGGCGATGCAGTCT-3'

TF101: 5'-ATGGAGAAGCTGATGC-3'

TFL112i: 5'-AATTTATCTTTTAACTCCAGAGGTTACCAATTC-3'

TF101: 5'-ATGGAGAAGCTGATGC-3'

TF122: 5'-AAATCTGCAGTGGTTGA-3'

TF121: 5'-GGTATTTTGCTGTAGC-3'

TF132: 5'-CTGAAAGCGCCTGTG-3'

TF155: 5'-ACGGAAAGATAAGGAAGCTTGCGT-3'

TFL166: 5'-TGTGTCATCTCTGAACAGAAGGTCCTTGGT-3'

Table 2. Comparison of nucleotide sequences in the transferrin gene

\begin{tabular}{llll}
\hline & Codon 247 & Codon 326 & Codon 394 \\
\hline Normal $^{\mathrm{a}}$ & CTA (L) & CCA (P) & GAA (E) \\
Normal $^{\mathrm{b}}$ & CTG (L) & CCA (P) & GAA (E) \\
Proband & TTG (L)/CTG (L) & CCC (P)/CCC (P) & GAA (E)/AAA (K) \\
Father & CTG (L)/CTG (L) & CCC (P)/CCC (P) & GAA (E)/AAA (K) \\
Mother & TTG (L)/CTG (L) & CCC (P)/CCC (P) & GAA (E)/GAA (E) \\
Normal & CTG (L)/CTG (L) & CCC (P)/CCC (P) & GAA (E)/GAA (E) \\
\hline
\end{tabular}

Amino acids encoded by codons are shown in parentheses

The initiation codon was defined as codon one

${ }^{a}$ cDNA sequence reported by Yang et al. 1984

${ }^{\mathrm{b}}$ cDNA sequence reported by Park et al. 1985

were heterozygous for CTG and TTG. The nucleotide polymorphism does not cause variation in the amino acid; CTA, CTG, and TTG all encode leucine. Codon 326 (amino acid residue 307) in exon 8 was found to be CCA, encoding proline, by both Yang et al. (1984) and Park et al. (1985). The patient and his parents were homozygous for CCC. The substitution of CCA to CCC is also synonymous.

In both of the previously reported sequences, codon 394 (amino acid residue 375) in exon 9 was GAA, encoding glutamate. Among the family members analyzed, the mother was also homozygous for GAA. On the other hand, the patient and his father were heterozygous for GAA and AAA. Figure 1 shows the results of the sequencing analysis of the paternal allele of the patient's $T F$ gene around codon 394. These results indicate that the patient inherited the $T F$ allele with AAA at codon 394 from his father. The substitution GAA to AAA causes a non-conservative amino acid change, glutamate to lysine. We examined a genomic DNA 
$3^{\prime}$

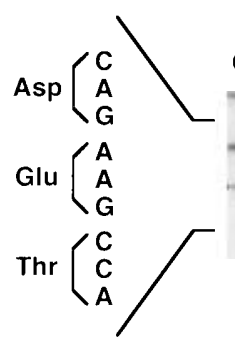

$5^{\prime}$ $3^{\prime}$

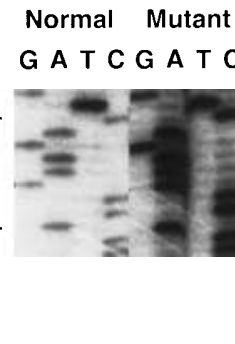

Fig. 1. Sequence analysis of the normal control and the paternal mutant allele of the patient. Nucleotide sequence ladders around codon 394 of the transferrin $(T F)$ gene are shown

sample of the patient's niece, his sister's daughter, and found that she was also heterozygous for GAA and AAA.

To investigate whether the $T F$ gene with AAA at codon 394 was distributed among the normal population, we examined DNA samples of healthy Japanese individuals by using a PCR-RFLP analysis. Since the sequence GAA at codon 394 lies in an MboII site, GAAGA, a genomic region containing a mutated codon AAA at that site is detectable by inspecting the MboII digested pattern. We amplified a 127-bp genomic DNA fragment including codon 394 of the $T F$ gene. In contrast to the normal GAA sequence at codon 394 giving rise to two $\mathrm{MboII}$ fragments $84 \mathrm{bp}$ and $43 \mathrm{bp}$ long, the mutated codon AAA gave a single undigested fragment. The results of the digested amplified DNA of the patient and his family are shown in Fig. 2. Consistent with the results of the sequencing analysis, the patient, his father, and his niece showed a heterozygotic RFLP pattern. The mother and two healthy controls showed a mono- or homozygotic normal pattern. Genomic DNA samples from 100 unrelated healthy Japanese individuals were examined, and all were homozygous for GAA at codon 394. Therefore, the $T F$ gene with AAA at codon 394 is unique to the allele that the patient inherited from his father.

To assess whether the amino acid substitution, from glutamate to lysine at amino acid residue 375 , affects the structure of the TF protein, we predicted the secondary structure of the normal and mutant TF proteins by using the method reported by Chou and Fasman (1978). The mutant $\mathrm{TF}$ with lysine at amino acid residue 375 was predicted to have an additional turn structure in a coiled region of the carboxyl-terminal iron-binding lobe.

\section{Discussion}

Our previous isoelectric focusing analysis of serum samples of the proband and his family suggested that the proband carried heterozgotic $T F$ alleles. The paternal allele expressed abnormal TF with a more basic isoelectric focusing

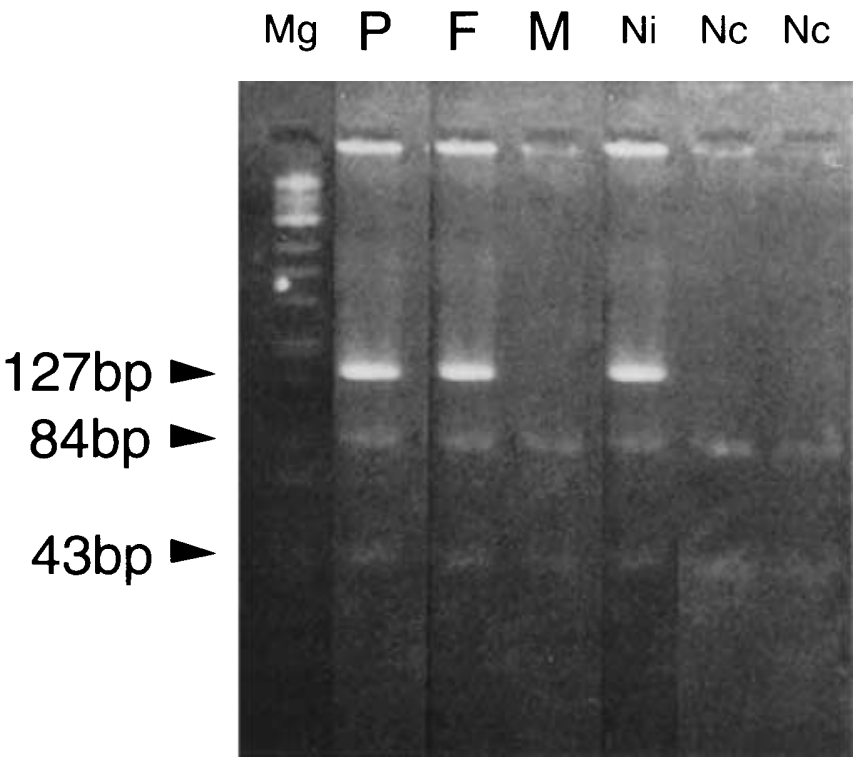

Fig. 2. Detection of the GAA to AAA mutation at codon 394 by the polymerase chain reaction-restriction fragment length polymorphism (PCR-RFLP) method. A genomic DNA fragment $127 \mathrm{bp}$ in length including codon 394 of the $T F$ gene was amplified and digested with MboII, which recognizes GAAGA. The GAA to AAA mutation at codon 394 disrupts the $M b o$ II site. The normal allele shows an $84-b p$ and a 43-bp band after MboII digestion. The PCR product originated from the mutant allele was not recognized by the restriction endonuclease and shows only a 127-bp band. Genomic DNA samples from the patient, his parents, his niece, and two normal control individuals were analyzed. Lane $\mathrm{Mg}$, DNA size marker; lane $P$, patient; lane $F$, father; lane $M$, mother; lane $N i$, niece; lanes $N c$, normal controls

point than that of normal TF, and the maternal allel expressed no TF (Hayashi et al. 1993). The results of the DNA sequence analysis of the present study are consistent with the results of the previous protein analysis. The GAA to AAA transition at codon 394 observed in the paternal allele of the $T F$ gene is compatible with the abnormally basic isoelectric point of the mutant TF protein. The nucleotide substitution changes amino acid residue 375 from an acidic amino acid, glutamate $(\mathrm{pK}=4.3)$, to a basic amino acid, lysine $(\mathrm{pK}=10.5)$. The TF protein is composed of two lobes, each of which binds to one iron atom (Gorinsky et al. 1979). The change in amino acid residue 375 is predicted to make an additional turn structure in the carboxyl-terminal lobe. Although amino acid residue 375 is not located at any of the six positions directly involved in iron binding (Baker et al. 1992), the amino acid substitution in this patient may affect the iron-binding capacity or stability of this protein.

No mutation in the maternal null $T F$ gene was found in the entire protein coding region or the exon-intron boundaries. An abnormality in the transcription or stability of its mRNA might be the cause of the absence of the the maternal allele-derived TF protein. One possibility is that the seemingly silent substitution CTA/G to TTG at codon 247 affects the stability of its mRNA.

Recently, Beutler et al. (2000) reported the analysis of another patient with hereditary atransferrinemia. That patient was also a compound heterozygote: a maternal mutant 
allele carried a frameshift mutation resulting in a truncated TF protein, and a paternal mutant allele encoded mutated TF with a substitution of alanine at amino acid 477 to proline.

To directly prove that the mutations found in the patient in our study and that in the study by Beutler et al. (2000) were indeed the cause of the clinical abnormalities, a functional analysis with a recombinant mutant TF protein is called for.

Acknowledgments We are grateful to Dr. Yoshinao Wada (Osaka Medical Center and Research Institute for Maternal and Child Health) for providing some of the PCR primers used in this study. We also thank Dr. Kazuyuki Hamaguchi (Oita Medical University) and Dr. Satoru Senju (Kumamoto University) for helpful suggestions. The work was supported in part by Grants for Specific Disease from the Ministry of Health and Welfare of Japan. The authors also wish to acknowledge the language assistance of Mr. Brian Quinn.

\section{References}

Baker ED, Baker HM, Smith CA, Stebbins MR, Kahn M, Hellstrom KE, Hellstrom I (1992) Human melanotransferrin (p97) has only functional iron-binding site. FEBS Lett 298:215-218

Beutler E, Gelbart T, Lee P, Trevino R, Fernandez MA, Fairbanks VF (2000) Molecular characterization of a case of atransferrinemia. Blood 96:4071-4074

Cáp J, Lehotská V, Mayerová A (1968) Congenital atransferrnemia in a 11-month-old child (in Czech). Cesk Pediatr 23:1020-1025

Chou PY, Fasman GD (1978) Prediction of the secondary structure of proteins from their amino acid sequence. Adv Enzymol 47:145148

Gorinsky B, Horsburgh C, Lindley PF, Mors DS, Parkar M, Watson JL (1979) Evidence for the bilobal nature of diferric rabbit plasma transferrin. Nature 281:157-158
Goya N, Miyazaki S, Kodate S, Ushio B (1972) A family of congenital atransferrinemia. Blood 40:239-245

Hamil RL, Woods JC, Cook BA (1991) Congenital atransferrinemia: a case report and review of the literature. J Clin Pathol 96:215218

Hayashi A, Wada Y, Suzuki T, Shimizu A (1993) Studies on familial hypotransferrinemia: unique clinical course and molecular pathology. Am J Hum Genet 53:201-213

Heilmeyer L, Keller W, Vivell O, Keiderling W, Betke K, Wöhler F, Schultze HE (1961) Kongenitale Atransferrinemie bei einem sieben Jahre alten Kind. Dtsch Med Wochenschr 86:1745-1751

Loperena L, Dorantes S, Medrano E, Berrón R, Vega L, Cuarón A, Rodríguez C (1974) Atransferrinemia hereditaria. Bol Med Hosp Infant Mex 31:519-535

Morgan EH (1983) Synthesis and secretion of transferrin. Academic, New York, pp 331-355

Park I, Schaeffer E, Sidoli A, Baralle FE, Cohen GN, Zakin MM (1985) Organization of the human transferrin gene: direct evidence that it originated by gene duplication. Proc Natl Acad Sci U S A 82:3149-3153

Ponka P, Schulman HM (1985) Acquisition of iron from transferrin regulates reticulocyte heme synthesis. J Biol Chem 260:1471714721

Ritchie RF (1975) Automated immunoprecipitation analysis of serum proteins. In: Putnam FW (ed) The plasma proteins: structure, function, and genetic control. Academic, New York, pp 375-425

Sakata T (1969) A case of congenital atransferrinemia (in Japanese). J Pediatr Pract 32:1523-1528

Sambrook J, Fritsch EF, Maniatis T (1989) Isolation of DNA from mammalian cells: Protocol I. In: Molecular cloning. A laboratory manual, 2nd edn. Cold Spring Harbor Laboratory Press, Cold Spring Harbor, New York, pp 9.16-9.21

Schaeffer E, Lucero MA, Jeltsch JM, Py MC, Levin MJ, Chambon P, Cohen GN, Zakin MM (1987) Complete structure of the human transferrin gene: comparison with analogous chicken gene and human pseudogene. Gene 56:109-116

Walbaum R (1971) Déficit congénital en transferrine. Lille Méd 16:1122-1124

Yang F, Lum JB, Mcgill J R, Moore CM, Naylor SL, van Bragt PH, Baldwin WD, Bowman BH (1984) Human transferrin: cDNA characterization and chromosomal localization. Proc Natl Acad Sci U S A 81:2752-2756 